\title{
Obtaining Design Requirements from the Public Understanding of Driverless Technology
}

\author{
Patrick Langdon ${ }^{1}$, Ioannis Politis ${ }^{1}$, Mike Bradley ${ }^{1}$, \\ Lee Skrypchuk ${ }^{2}$, Alexander Mouzakitis², and John Clarkson ${ }^{1}$ \\ ${ }^{1}$ Engineering Design Centre, Department of Engineering, University of Cambridge, \\ Trumpington Street, Cambridge, CB2 1PZ, UK \\ \{pml24, ip332, mdb54, pjc10\}@cam.ac.uk \\ ${ }^{3}$ Jaguar Land Rover, Research \& Technology, Coventry, United Kingdom \{LSKRYPCH, \\ AMOUZAK1\}@jaguarlandrover.com
}

\begin{abstract}
Despite a large literature on autonomous vehicles and a large number of relevant surveys examining public perception of self-driving vehicles, recent studies suggest that reported public perception is rapidly changing, volatile, and highly dependent on context and potential biases in reporting. In addition, some conclusions from very recent studies are supported: namely, that very little of the survey literature addresses key user centred design consideration that arise from inclusion of the wider population in the use of autonomous vehicles. We report initial studies that are intended to form part of a method currently under development that aims to bring together automotive design and Inclusive design; an analytical functional approach to design for user capability in the wider population. This paper describes the development stages of a user centred design capable methodology for sampling public opinion from a range of different qualitative studies. The finding are made available at two levels. Firstly, a general report on public perceptions', examines key elements of sensitivity in responses to autonomous vehicle issues. Secondly, the methodology shows how to address in an unbiased way the specification and design a number of potential HMI concepts for managing takeover from car to driver in an autonomously capable vehicle in urban and highway usage cases.
\end{abstract}

Keywords: Human Factors, Inclusive Design, Surveys, Autonomous cars; Public opinion

\section{Background and Motivation}

Although there is a burgeoning and long standing literature on Self Driving Cars (SDC) and their effect on society [4] a good deal of this literature has been carried out within the domain of the research lab, or specialists in socio economics, transport researchers, or Business information [3] reporting.

Older survey studies have reported predictions regarding the prevalence of specific technologies that were found to be highly conservative. Recent studies [19; 22;15;1 ;4] suggest that reported public perception is divided, rapidly changing, and highly 
dependent on context and potential biases in reporting [3]. Hence, older survey and laboratory studies are likely to be less informative about current public opinion due to the effect of public exposure to publicity. This has come from various sources, including OEMs, advertising, the provision and advertising of automated and autonomous feature in new market offerings and a number of new initiatives aimed at putting autonomous vehicles onto UK roads (Venturer, UK Autodrive)[23; 24] and their surrounding media comment.

There are, however, a number of very recent reviews of public opinion and social implications of attitudes towards autonomous vehicles. Kyriakidis, Happee \& de Winter [12] presented the most recent academic review of public opinion and report on a 5000 person study carried out in 109 countries in 2015. Noting the diversity of responses, they found that manual driving was preferred and that $33 \%$ thought full automation likely to be enjoyable. However, most concern was about hacking, legal and safety issues, at different levels of sophistication depending on demography. Respondents saw high automation as premium product worth in excess $\$ 30,000$, which appears to reflect a specific view of technology as the domain of the high earning bracket.

A very recent study carried out in the UCL Transport Institute report for the Department of Transport [3], reports that urban men more likely to be interested in AV than women and that older people were less likely to be interested in AV, in general. They found that interest varied with Geographical area and that those enjoying driving less were more likely to be interested in the possibilities of AVs. The study identified three key factors in the public perception of AVs :

1. Safety and cybersecurity

2. Perceived usefulness and benefits

3. Cost (should be less than conventional cars)

These themes summarise the main considerations reported by many studies and were well matched to the present thematic analysis, taking safety and security to be effectively "trust". These themes can also be seen repeatedly in a number of other recent studies.

The THINKGOODMOBILITY 2016 [22], survey of Autonomous Vehicles sampled 12000 respondents and 48 people in focus groups in four countries. They found that $44 \%$ were uncomfortable with AVs, or in sharing the road with AV (41\%). When asked about activities people expected to do while autonomous, $42 \%$ stated text, $37 \%$ internet, $28 \%$ reading, $27 \%$ e-mail, $19 \%$ sleep, and $18 \%$ video.

Of the whole sample $43 \%$ felt that AV were safer than driving and $19 \%$ did not, with the remainder (62\%) neutral. This large neutral figure may be a characteristic of recent sampling in surveys as around $60 \%$ stated that AVs did not know enough to judge. They also felt that AVs did not have enough "common sense" for a human driver mix. The majority, 73\%, thought AVs could malfunction, and 70-80\% thought that the driver should be in control and should have access to a steering wheel. A large response, 82\% preferred to be aware of the road at all times.

The very recent Venturer Project carried out a survey based on understanding the socioeconomic adoption scenarios for autonomous vehicles based on key concepts from Socio-technical regimes [6] and Technology Acceptance models (Legris, 2003) [14].Aimed at level of awareness and general attitudes to AV, they made a number of findings summarised here. They found a clear awareness of AV and generally positive 
expectations of their benefits. The driving task was still enjoyed and manual control was still seen positively, while AVs were associated with a loss of control. There were doubts over safety but agreement that benefits may be in boring, undemanding driving, not complex urban manoeuvring.

Currently therefore, despite many surveys with comparable findings, recent studies suggest that it is not clear what the public understanding may be of driverless technology and how it is perceived as part of their lives in the near and middle future time scales. Very little work has focused on the totality of the issue from the users' perspective, which concerns the specific technology, and extends into both the likely users in society and, importantly, what journeys they make. This will be related to peoples' usage of vehicles, the journeys they make, and the relationship between these and the lifestyles they are able to lead in their home locations. Their perceptions may well be quite distinct from that of technology developers, governance and service planners. Very little rigorous research has been devoted to the task of identifying potential users' understanding, opinions, needs, and preferences for the specifics of new generation of autonomous vehicle technology. In particular, there is a need to understand how effective human machine interfaces (HMI) can be designed to best provide the driver safe control when either handing control to the car or taking control back from the car in urban and highway driving situations [18]. This may be the key to establishing technological acceptance in both driving and non-driving elements of the population.

\section{Methodology}

The collected data was essentially qualitative and hence methodology was driven by the principles of qualitative research. For this to be effective, a range of sources was necessary using differing methods such as interviews, focus groups, surveys and field studies. Each of these sources were fully documented; fully referenced and described, and human data taken with Video and Audio recordings and transcription of interactions. The outcomes in Initial interviews, focus groups and research were followed by an interpretation and consolidation stage where a triangulated interpretation was formed taking into account all the data sources [5; 7]. All sources of data and the extent of their agreement and disagreement were decided, moderated by the methodological strength of their origins. For example, survey data was compared for agreement with thematic analysis but themes common only to one were included or abandoned depending on the strength of the data collection method. It was necessary to use a compressed format for the outcomes of the triangulated interpretation stage. A convenient representation of this sort was a concept or requirements table. In addition, once the Thematic Analysis for both Interviews and Focus Groups had been completed, interpretation of the resulting themes provides a set of key insights, and these are reported.

The project addressed the problem through a human centered design approach using qualitative research techniques $[2 ; 5 ; 7]$. The analysis of transcribed data was made using standard social science techniques to analyse the data using qualitative research approaches; such as that of Grounded Theory [7]. A general qualitative design tools 
NVIVO[16], yielding a thematic analysis used during different stages of interpretation. The method followed the plan shown in Figure 1.

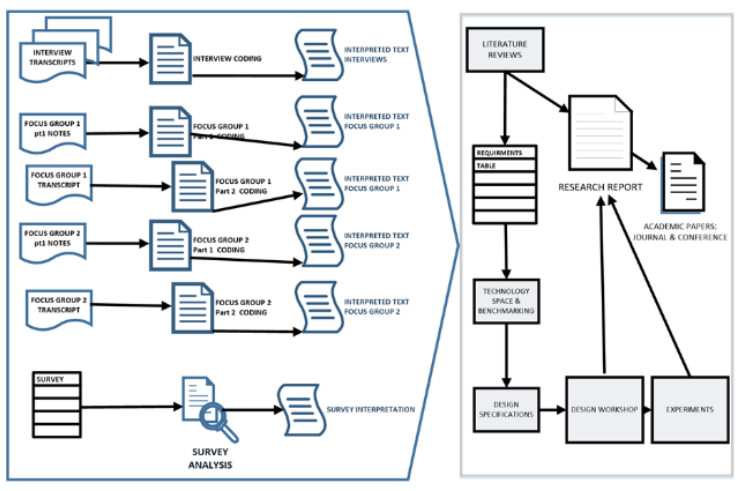

Figure 1 - Overview of methodology plan. Other aspects; technology review, Focus groups and the design workshop stage are reported elsewhere [Ibid Politis et al]

Interview participants were sampled to cover an appropriate range of the population that it is intended to generalise the results to. Some criteria for choice were established based on age, gender, demographic group and geographical region and these were matched to the traveller needs survey; described below, on a quota basis. No respondents with strong impairments of vision, physical or hearing capability were used and the sampling was chosen to include, young, late middle-aged males and females from metropolitan and rural areas. The sampling aim was also to achieve inclusion in functional capability, age, gender, social economic status and geographical location. This was achieved by using Inclusive design criteria such as those illustrated in Figure 2. The field of inclusive design relates the capabilities of the population to the design of products by better characterising the user-product relationship. As such, its advantage is that of inclusion of functionality for varying capability into mainstream society. Inclusion refers to the quantitative relationship between the demand made by design features and the capability ranges of users who may be excluded from use of the product because of those features. Capability ranges were characterised by Functional analytical scales that can be numerically compared to a product function feature analysis [11]. The primary aim of inclusive design is to make mainstream products and services accessible to, and usable by, as many people as reasonably possible [10]. Designing inclusively ensures that the needs of user groups that are often underrepresented in the design process are accounted for. This process not only can facilitate a quantification of the baseline inclusion, but also informs the design process and provides a metric to evaluate new design concepts [13]. The benefit of using an inclusive user group lies in the fact that a wide spectrum of capabilities is taken into consideration in the design phase. These capabilities can be auditory, visual, cognitive, or related to dexterity and mobility. Considering an inclusive sample of users with a variety of such capabilities results in designs that can provide a satisfactory user experience not only for the population with capability limitations, but also for a larger proportion of the population [13]. 

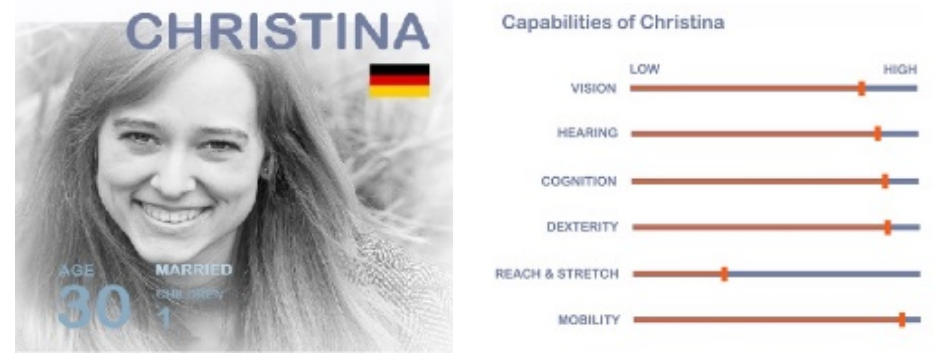

Figure 2 - Inclusive design persona profile showing functional scales [11]

As basis for sampling that took into account the journeys and personas of users, a source of information was the accurate National Survey data. (Figure 3) the Intelligent Mobility, Traveller Needs and UK Capability Study (2015), an recent survey of UK journeys and transportation usage with 10,000 respondents in the UK, and enabled the formation of sampling categories of “persona-journeys”. Hence potential users were classified by the journey types they undertake [8] For example:

'Dependent Passengers' covers 21\% of UK population and 18\% of journeys. It is a segment that is dependent on others to meet its mobility needs. This traveller type consists of a number of groups, such as young people (who typically get driven by their parents), elderly people, and travellers with impairments. They take a majority of their journeys as car passengers and the remainder is typically covered by either bus or by walking, with journeys split representatively between work and leisure.

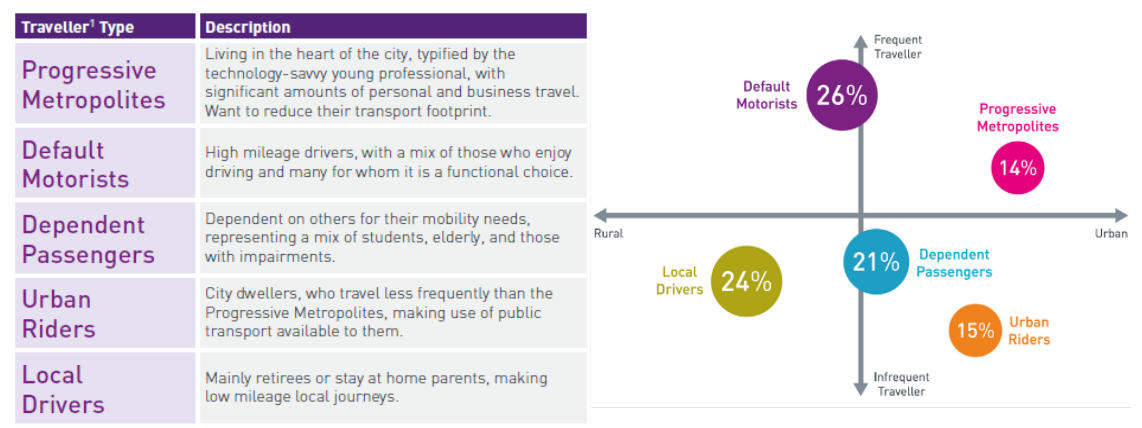

Figure 3 - User journey personas (top-level) identified by the IM UK Traveller Needs Survey

Other aspects of the method; technology benchmarking, the Focus groups and the design workshop stage, are reported elsewhere. The relevant literature sampled issues concerning autonomous vehicle takeover including: Automotive research papers, trade journals and press releases. 


\subsection{Semi-structured interviews}

The outcomes of several stages of the qualitative research are reported: The initial public interviews with representative of groups identified by the Traveler needs survey. A total of eight semi-structured interviews were carried out aiming to obtain a cross-section of opinions and preferences from interviews based on the questioning regarding the appearance, function and use of future autonomous vehicles without any introduced biases of either explanation or suggestion regarding the HMI technology involved. Exposure to autonomous technology was based on two examples of current state of the art offerings represented by commercial Public Relations videos:

1) Video 1 OEM concept autonomous interior video:

2) Video 2 OEM video of fully autonomous car:

The transcribed interviews were analysed using thematic analysis software (Nvivo v.11). Two coders' themes were separately developed then compared and merged on the basis of content and statements by the respondents, the number and strength of the mentions, and the commonality with the other coder. The merging was made with the reduction of themes where repetitive or the reclassification of individual personal themes by merging them with a larger theme. New themes were added to re-classify idiosyncratic statements and some small themes were deleted if they were no longer significant in the new scheme.

\section{$2.2 \quad$ Two focus groups}

These were carried out addressing the issues of the understanding of autonomous vehicle takeover process and the HMI technology that may be involved; (reported in this volume IBID). An assessment tool (e.g. a survey) identifying the participants' demographics and initial attitudes towards the design topic in order to recruiting representative users. This identified detailed user requirements for concept designs. The participants to the focus group were: technically minded individuals, in one case, and: inclusive group with a range of ages and gender to represent the desired user group for the design. A series of focus groups with different participant sets can provide a wider set of outputs, which were then triangulated into the interpretation process. The focus groups were video recorded and transcribed, and analysis of the script and video recordings is given in (Ibid, Politis et al, 2017). Facilitation had a pre-agreed structure, which enabled dialogue between participants while audio-visual tools display the focus points of discussion, in this case road layout diagrams and toy cars. The facilitator's role was to trigger continued discussion without predisposing the participants to any particular philosophy and which were kept technology and issue neutral to HMI in relation to the primary questions of takeover.

\subsection{The interpreted outcomes analysis}

The interpreted outcomes analysis took as input the interpreted findings of these previous stages of the research. Hence, and following Goodman-Deane et al [7] the analysis was made using modified qualitative research approaches, such as that of Grounded Theory. This yielded a thematic analysis with an accompanying interpretation by the researchers. Importantly, the analysis requires a continued process 
of coding during the theme development. Themes were recoded and monitored iteratively. Multiple coders, without interaction, compared their coding's and converged onto a final set. Themes from initial sources were merged using a triangulated approach to generate a definitive interpretation and representative examples of the theme, and a rationale for it. A descriptive analysis was undertaken with the main themes described as a hierarchical list (See, for example in Figure 4). A number of top level item themes subsumed sub-themes, as shown. For example, "HMI Issues" subsumed a number of themes, including "Algorithm expectations" and "Distractions". A detailed interpretation was then made in text format, breaking down each theme following an overview of the entire theme

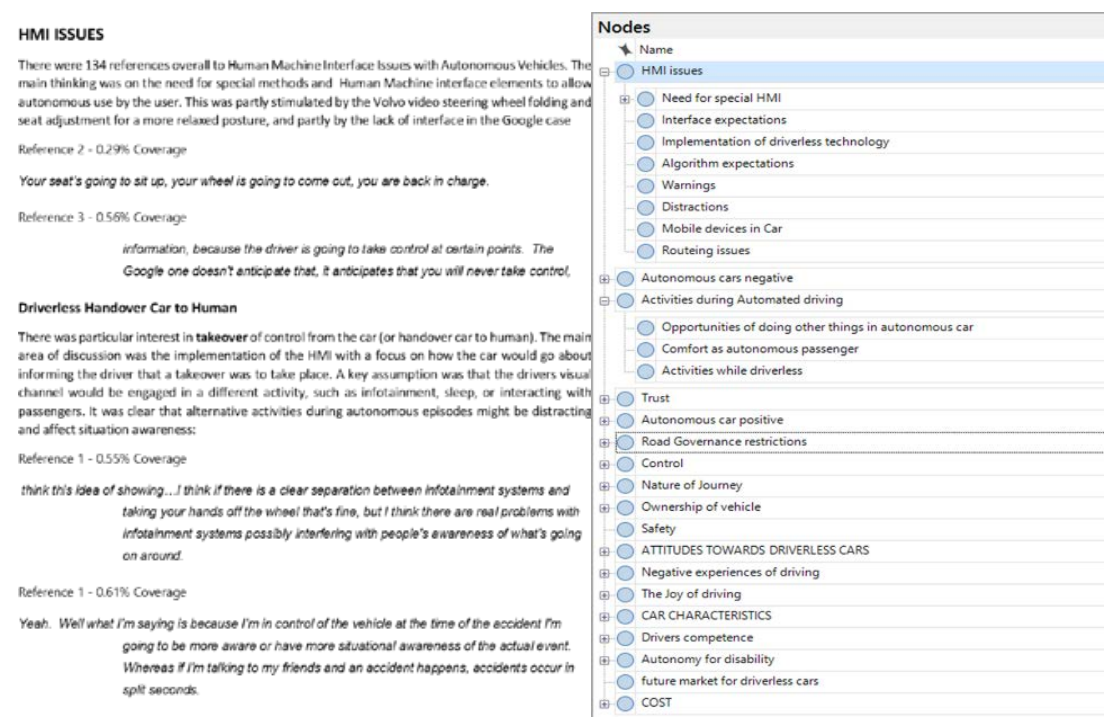

Figure 4 - (left) Example of the interpreted analysis the top-level thematic nodes organized by number of references in the 8 sources. On the right is the themes resulting from the various stages of recoding, including hierarchies where they were apparent.

\subsection{The concepts and requirements insights}

This table summarised the interpreted findings. The interpretation of the final themes forms a large corpus of text even with a second stage of recoding. One purpose of the interpreted analysis was to form identified micro-concepts or insights directly related to the issues identified and used as input to the themes. These were, by their nature repetitive and convergent. However, an alternative representation was used that allowed the comparison of the numerous occurrences in the interpretation and that was also useful as input to the Design process. The representation used was the engineering design requirements table, modified for social science requirements and used as a representation that captured the richness of the interpreted findings. It had the advantage of being a compressed format for the outcomes of the triangulated interpretation stage, especially but allows human centred design requirements to be generated. At a 
minimum this gave each captured entity a unique identifier for later referencing; a name, a description, and a rationale. In principal, this allowed inclusive requirements to be generated for input into the design stages and final concepts. A convenient representation of this sort can be a requirements table that enumerates each ideaconcept from the interpreted thematic analysis, gives it a description, a rationale for function and indication of its application in a design, including whether it is mandatory or optional. Such a table was constructed for the analysis from the two levels of interpreted findings of the interviews, focus groups and literature reviews. An initial table with all insights included a large number of insights, including repetitions and hierarchically related constructs, which were removed to reduce the total number.

Output insights from the previous process were used both as input in to the HMI concept development stages and also formed a stand-alone set for wider interpretation. For this purpose, insights were recoded into graphic card format and a complete set recorded for further analysis. A sub-set of these were generated, ignoring repetition or duplication of theme insight and context and this smaller set was used in the design workshops. This set is exemplified here, as a short example list. The full list contains 96 items and is the basis for further work.

Table 1 Examples of insights, the result of triangulated interpreted qualitative data from interviews, surveys and focus groups.

\begin{tabular}{|c|c|}
\hline Identifier & Decription \\
\hline INT1: Multimodality & $\begin{array}{l}\text { In addition to a visual, there should be a series of } \\
\text { increasingly strident signals as the takeover point approaches. }\end{array}$ \\
\hline $\begin{array}{l}\text { INT14: Response to } \\
\text { Inaction from Driver }\end{array}$ & Car adopts safest strategy \\
\hline $\begin{array}{l}\text { INT8: System } \\
\text { Capability }\end{array}$ & The system should simple and easy to understand. \\
\hline $\begin{array}{l}\text { FC50: Countdown to } \\
\text { Takeover }\end{array}$ & $\begin{array}{l}\text { Seen as similar to Satnav countdown instructions to a } \\
\text { junction }\end{array}$ \\
\hline $\begin{array}{l}\text { INT12: Warning by } \\
\text { Changing Ambient } \\
\text { Interior Configuration }\end{array}$ & Advantages of interior ambience were clear \\
\hline $\begin{array}{c}\text { FC6: Integration of } \\
\text { Autonomy with Traffic }\end{array}$ & $\begin{array}{l}\text { Uncertainty how autonomous cars can perform alongside } \\
\text { conventionally driven vehicles }\end{array}$ \\
\hline ID29: Time Recovery & Facilities for activities during autonomous driving \\
\hline $\begin{array}{l}\text { ID33: Impairment } \\
\text { Compensation }\end{array}$ & Provide inclusive design \\
\hline INT17: Regain Control & Driver should be able to take control of car \\
\hline
\end{tabular}




\section{$3 \quad$ Discussion and Conclusions}

A number of insights were distilled from the qualitative data collected from a series of interviews and focus groups, These were triangulated and interpreted in the light of a number of sources of data, including technology benchmarking and recent survey findings on attitudes to a social and economic issues associated with the introduction of autonomous vehicles technology at Level 3 and also Level 4.

Some manufacturers have autonomous cars on the roads effectively already functioning at SAE Automation level 3 [18] where the vehicle takes control of both steering and acceleration in specific circumstances but the driver must monitor the vehicles progress, ready to intervene to takeover control. Considerable media attention has been directed at accidents and incidents involving these Level 3 vehicles as a response based on public safety. Level 5 vehicles also represented in test form on UK roads and these require on intervention by the driver as they can handle the entire dynamic driving task [18].

In their thematic analysis of previous work and their own survey data, Cavoli at al, [3] draw wide ranging conclusions. However, they also identify a clear pathway for concern based on their extensive thematic review on Social and behavioural questions associated with automated vehicles that concerns the design of autonomous takeover technology and its HMI (Figure 5). In particular, it would appear that the general perception is that engagement with non-driving tasks at Level 3 will introduce potentially unsafe situations leading to traffic incidents. These then may affect the uptake and acceptance of the technology as public perception is affected.

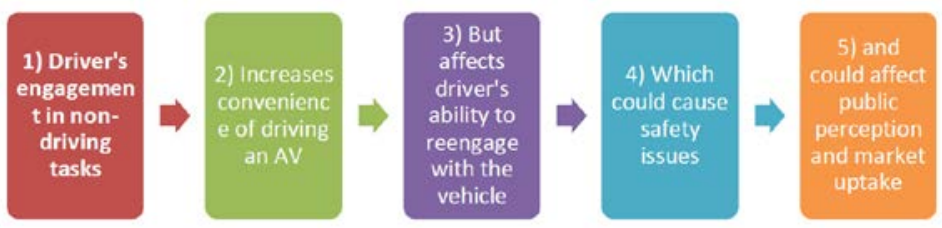

Figure 5 The UCL Transport Institute report for the Department of Transport: Potential safety risks in cases of partially or highly automated AVs

Despite the general finding that around 33\% of sampled populations are generally positive towards AV technology, a considerable number of studies report 30\%-50\% that are either neutral or have no view regarding the technology. These participants are presumably related to the Mainstream and Price-drop” groupings of the Technology Acceptance Models [Legris] and their neutrality may arise from lack of knowledge of technology, or reluctance to commit unless further evidence from media and personal experience is forthcoming. This result was common to all the recent studies reviewed. On the other hand, a solid proportion of around $25 \%$ are reluctant to hand over control, would retain awareness of the road and see the AV driving environment as potentially dangerous and stressful. Again, it is likely that these may represent the "Last to Adopt" and "Technology Avoiders" groups of such models, who resist technology offerings. Interestingly, the focus group findings reported here (e.g. FC6: Integration of Autonomy with Traffic; and INT17: Regain Control) suggest that these negative 
attitudes can be associated with technology sophisticated urban and suburban dwellers who appreciate the potential failings of engineering [12;22]; rather than those who rely on blind trust in technology.

In addition to this, a number of conclusions made in the Cavoli study indicate that this field is far from understood. For example, they state that it will be necessary to further understand older people's perception of AVs and their potential reluctance to use the technology and also, importantly, that further research is needed to assess nondrivers' perception and expectations of AVs, and why a large percentage of females seem less interested in AVs compared to men. Nevertheless, they also found that $42 \%$ would currently force their car into a traffic stream, as a measure of driving sociability. Higher technology respondents showed more openness to technology and were more open to AVs. The least open to AV's were found to be more sociable drivers with low technology understanding but high levels of optimism, indication a form of "blindfaith" acceptance.

During the THINKMOBILITY survey there was a reported $\sim 6 \%$ improvement of attitude as a result of social exposure to AV material suggesting that attitudes are changing rapidly in the UK and internationally. The Venturer Project survey [4] found that, in reviewing considerable body of previous literature, that variability in responses may have been due to response biases in sampling, particularly for surveys. They concluded that if we are to understand the nature of public opinion in this area surveys were not sufficient. They propose that further collection of specific qualitative data will be needed based upon identified populations responding to more detailed usage and technology cases. The authors would concur, with the further specification of the importance on inclusive populations in sampled groups; catering for the wider nonmainstream population, including the older drivers and those whose capabilities may not be well matched to driving tasks. In addition, with reference to widening the usage of autonomous vehicles, the greater proportions of these groups are likely to be found among current non-drivers.

\section{Acknowledgements}

This work was funded by EPSRC and Jaguar-Land Rover, as part of the project Human Interaction: Designing Autonomy in Vehicles (HI:DAVe), Project Grant Number: EP/N011899/1.

\section{$4 \quad$ References}

[1] Begg, D. (2014). A 2050 vision for London: What are the implications of driverless transport? Retrieved from http://www.transporttimes.co.uk/Admin/uploads/64165-Transport-Times A2050-Vision-for-London_AWWEB-READY.pdf 
[2] Breakwell G M, Hammond, S., Fife-Schaw,C., (2000) Research Methods in psychology (2nd) SAGE.

[3] Cavoli, C. et al., 2017. Social and behavioural questions associated with Automated Vehicles. A Literature Review, London: Department for Transport.

[4] Clark, B., Parkhurst, G., Ricci, M.: Understanding the socioeconomic adoption scenarios for autonomous vehicles: A literature review. , UWE, Bristol (2016).

[5] Flick, U.: An Introduction to Qualitative Research - Fourth Edition. SAGE (2009).

[6] Geels, F. (2005) The Dynamics of Transitions in Socio-technical Systems: A Multi-level Analysis of the Transition Pathway from Horse-drawn Carriages to Automobiles (1860-1930), Technology Analysis and Strategic Management, 17(4), 445-476.

[7] Goodman-Deane, J., Langdon, P., and Clarkson, J., (2010). Key Influences on the User-Centred Design Process. Journal of Engineering Design 21(2-3), pp. 345-373, April-June 2010.

[8] IMTNS , IM Travellers needs Survey, (2015)

[9] https://ts.catapult.org.uk/current-projects/traveller-needs-uk-capability-study/

[10] Ioannis Politis1, Patrick Langdon1, Mike Bradley, Lee Skrypchuk,, Alexander Mouzakitis, and John Clarkson (2017), Designing Autonomy in Cars:A Survey and Two Focus Groups on Driving Habits of an Inclusive User Group, and Group Attitudes Towards Autonomous Cars (ibid.)

[11] Keates, S, Clarkson P.J., (2004) Countering Design Exclusion - An Introduction to Inclusive Design. Springer-Verlag, London, UK.

[12] Kunur, M Langdon, P Bradley, M Bichard J-A Glazer, E Doran, F Clarkson, J Loeillet, J.: Creating Inclusive HMI Concepts for Future Cars Using Visual Scenario Storyboards Through Design Ethnography. In: International Conference on Universal Access in Human-Computer Interaction. pp. 139149. Springer (2015)

[13] Kyriakidis, M., Happee, R., De Winter, J.C.F.: Public opinion on automated driving: Results of an international questionnaire among 5000 respondents. Transp. Res. Part F Traffic Psychol. Behav. 32, 127-140 (2015).

[14] Langdon, P., and Thimbleby, H., (2010), Inclusion and Interaction: Designing Interaction for Inclusive Populations, Editorial for Special Edition of Interacting with Computers, V22, pp439-448, Elsevier. 
[15] Legris, P., Ingham, J. and Collerette, P., 2003. Why do people use information technology? A critical review of the technology acceptance model. Information \& management, 40(3), pp.191-204.

[16] Missel, J. (2014). Ipsos MORI Loyalty Automotive Survey. Retrieved from http://www.ipsosmori.com/researchpublications/researcharchive/3427/Only18-per-cent-of-Britons-believe-driverless-cars-to-bean-importantdevelopment-for-the-car-industry-to-focuson. aspx?utm_campaign=cmp_325684\&utm_source=getanewsletter

[17] Nvivo, (2017), http://www.qsrinternational.com/product, QSR International.

[18] Politis, I., Brewster, S., Pollick, F.: Language-Based Multimodal Displays for the Handover of Control in Autonomous Cars. In: Automotive UI 2015. pp. 3 10. ACM Press (2015).

[19] SAE,http://cyberlaw.stanford.edu/files/blogimages/LevelsofDrivingAutomatio n.pdf, accesed 27.02.17.

[20] Schoettle, B., Sivak, M.: A survey of public opinion about autonomous and self-driving vehicles in the US, the UK, and Australia. (2014).

[21] Schoettle, B., Sivak, M.: Motorists’ Preferences for Different Levels of Vehicle Automation: 2016. (2016).

[22] 11Sommer, K. (2013). Continental Mobility Study 2013

[23] Tennant,C., Howard, S., Franks, B., Stare, S., and Bauer, M.W., (2017), Autonomous Vehicles - Negotiating a Place on the Road: A study on how drivers feel about Interacting with Autonomous Vehicles on the road, London School of Economics and Political Science, Goodyear Europe, Middle East and Africa (EMEA.

[24] UK Autodrive: www.ukautodrive.com/

[25] Venturer: www.venturer-cars.com/ 